\title{
3D microfabrication of complex structures for biomedical applications via combination of subtractive/additive direct laser writing and 3D printing
}

\author{
Linas Jonušauskas ${ }^{1}$, Sima Rekštyte் ${ }^{2}$, Edvinas Skliutas ${ }^{3}$, Simas Butkus ${ }^{4}$, \\ Mangirdas Malinauskas ${ }^{5}$ \\ 1,2,3,4,5 Vilnius University, Lithuania \\ E-mails: ${ }^{1}$ linas.jon@gmail.com (corresponding author), ${ }^{2}$ sima.rekstyte@ gmail.com, \\ ${ }^{3}$ edvinas.skliutas@gmail.com, ${ }^{4}$ simas.butkus@ff.vu.lt, ${ }^{5}$ mangirdas.malinauskas@ff.vu.lt \\ (Received 1 March 2016; accepted 19 May 2016)
}

\begin{abstract}
In this work we present current progress on employing direct laser writing (DLW) for creation of 3D microstructures for biomedical applications. Both subtractive and additive variations of DLW allow fabricating structures for in vitro bioanalysis and in vivo tissue engineering. Furthermore, we show that efficiency of 3D microstructure manufacturing can be enhanced by combining femtosecond laser material processing with commercial 3D printers.
\end{abstract}

Keywords: laser microfabrication, biocompatible polymers, 3D biomedical structures.

\section{Introduction}

The prospect of manufacturing 3D microstructures that could be used in regenerative medicine and bioanalysis always generated high interest in the field of biomedicine. However, requirements for such objects are highly nontrivial. Materials have to be biocompatible, produced structures must have desired functional properties and high-throughput high-precision 3D fabrication technologies should be used.

An answer for such requirements could be direct laser writing (DLW). By applying ultrashort (from picosecond to femtosecond) laser pulses both subtractive and additive manufacturing is possible (Fig. 1) [1]. This enables combining a wide array of materials as well as freeform 3D geometry best suited for any given task. What is more, efficiency can be further enhanced by coupling laser fabrication with conventional 3D printing (3DP) technologies [2]. In such case fast $3 \mathrm{DP}$ is employed for the creation of the base structure, while the laser is used to further decorate it with fine features (from tens of $\mu \mathrm{m}$ to hundreds of $\mathrm{nm}$ [1]) that cannot be produced using $3 \mathrm{D}$ printing [2].

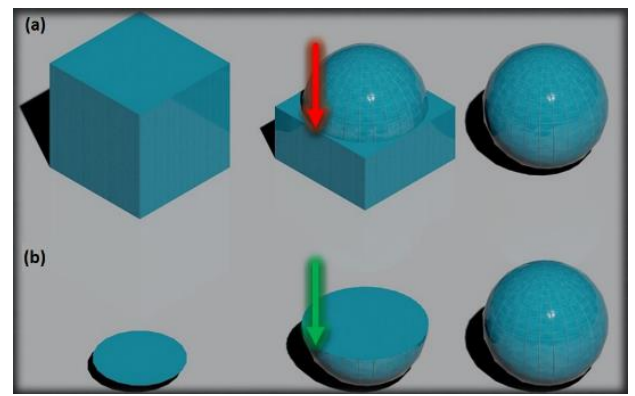

Fig. 1. Schematic representation of (a) subtractive and (b) additive fabrication [3]

Here we will discuss current progress on the aforementioned approach of combining subtractive and additive DLW with 3DP for the creation of structures that could be used in biomedical applications. Capabilities and problems arising when combining these technologies in

(C) 2016 The Authors. Published by VGTU Press. This is an open-access article distributed under the terms of the Creative Commons Attribution License (CC-BY 4.0), which permits unrestricted use, distribution, and reproduction in any medium, provided the original author and source are credited. 
practice will be explained. Examples of produced 3D microstructures such as scaffolds with defined pores/porosity or intertwined channels will be given.

\section{Methods}

Two high precision femtosecond laser "Pharos" (Light Conversion Ltd.) based fabrication systems were used. The first setup was tuned for subtractive sample processing by using higher light intensities ( hundreds of $\left.\mathrm{TW} / \mathrm{cm}^{2}\right)$ and first laser harmonic $(1030 \mathrm{~nm})$. Both cutting via sharp focusing or light filamentation can be realized with it (Fig. 2. (a)). The second one was applied in additive fashion for 3D microfabrication of the polymers and was based on the multiphoton lightmatter interaction (Fig. 2. (b)) [1]. Much lower light intensities $\left(\sim \mathrm{TW} / \mathrm{cm}^{2}\right)$ as well as II laser harmonic $(515 \mathrm{~nm})$ was applied in it. Details on these systems can be found in [4] and [5] respectively. Polymers used for the creation of the structures include but are not limited to hybrid organic-inorganic photopolymers (namely SZ2080), polylactic acid (PLA) and PEG-DA [2], including their formulation doped with metallic nanoparticles.

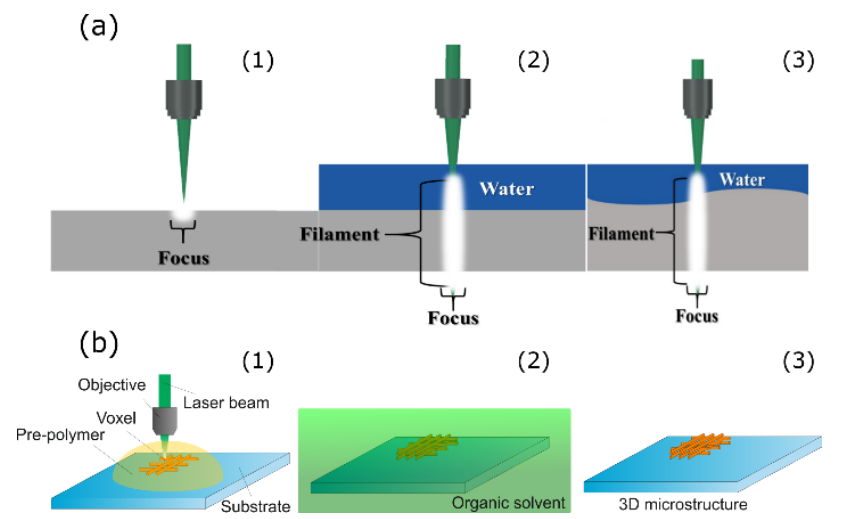

Fig. 2. (a) Schematics of cutting via sharp focusing (1) and via light filamentation (2) [2]. (3) - usage of light filaments also allows cutting when the sample surface is uneven [6]. (b) Multiphoton polymerization based 3D manufacturing: (1) fabrication, (2) development and (3) finished 3D microstructure [5]

Additionally two types of 3DP were tested. Applying either fused filament fabrication or stereolithography they offered different fabrication speeds (from $\mathrm{mm} / \mathrm{s}$ to $\mathrm{cm} / \mathrm{s}$ ), resolutions (from tens of $\mu \mathrm{m}$ to tens of $\mathrm{cm}$ ) and processable materials (thermocurable and photocurable).

\section{Results}

Microfluidical systems that could be used in bioanalysis or scaffolds for tissue engineering can be produced via the combination of 3DP and laser cutting. 3DP guarantees relatively high throughput fabrication of low spatial resolution structures. $\mathrm{Cm} / \mathrm{s}$ linear translation speeds result in fabrication durations in the range of minutes for $\mathrm{cm}$ sized microchannel systems and scaffolds. Laser cutting allows further functionalization of such objects by providing them with required shape and/or surface topography that cannot be produced with only 3DP (Fig. 3). 


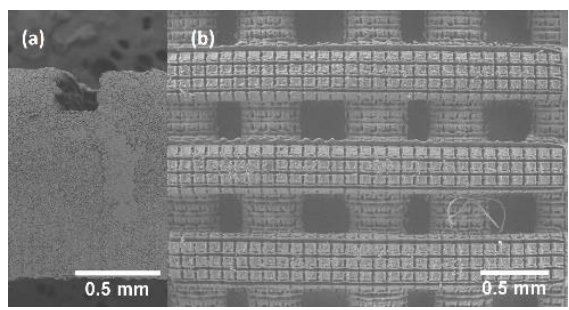

Fig. 3. Cross section of 3DP created microchannel that was afterwards cut via laser filamentation (a) [5]. (b) - enhanced topography of a 3DP scaffold via sharp focusing cutting [2]. Material - PLA

Additive fabrication via multiphoton absorption opens the possibility to produce complex true 3D microstructures with feature size as small as hundreds of nm [1]. Furthermore, composite manufacturing combining different materials in distinct parts of the structure can be realized this way [2]. Here we show that these capabilities can be used for the creation of 3D biomedical microobjects complex both in their architecture (Fig. 4 (a)) and materials used (Fig. 4. (b)). This is crucial for precise tunability needed in biomedical applications. Size of these structures can be from $\mu \mathrm{m}$ to $\mathrm{mm}$ in size with hundred $\mathrm{nm}$ resolution internal features. Shown objects can be manufactured in times ranging from minutes to hours depending on the complexity of internal geometry and overall volume (filling ratio) of the structure.

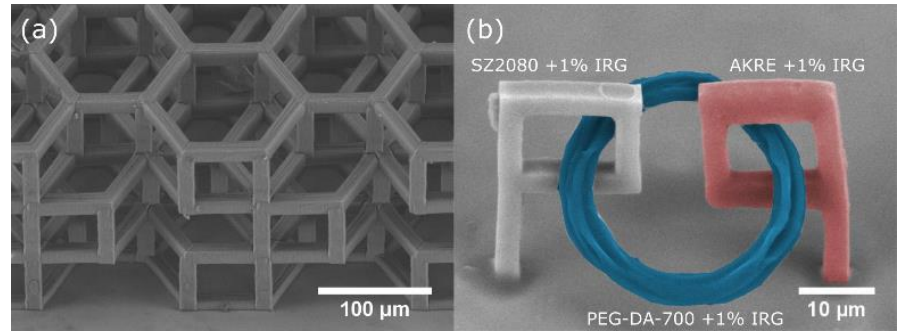

Fig. 4. (a) Complex shaped 3D scaffold for stem cell growth with hexagonal structure and poor size in range of tens of $\mu \mathrm{m}$. (b) Composite 3D microstructure created out of three different materials [2]

\section{Conclusions}

The presented results show how combination of sub-micrometre precision femtosecond laser material processing and rapid 3DP offers an efficient fabrication of complex 3D structures of biocompatible materials for applications in the fields of micro-analysis and tissue engineering.

\section{Acknowledgements}

We acknowledge ECs Seventh Framework Programme Laserlab-Europe VI JRA support BIOAPP (EC-GA 654148).

\section{References}

[1] Sugioka, K.; Cheng, Y. 2014. Femtosecond laser three-dimensional micro- and nanofabrication, Applied Physics Reviews 1(4): 041303. http://dx.doi.org/10.1063/1.4904320

[2] Malinauskas, M., et al. 2014. 3D microporous scaffolds manufactured via combination of fused filament fabrication and direct laser writing ablation, Micromachines 5(4): 839-858.

http://dx.doi.org/10.3390/mi5040839 
[3] Malinauskas, M., et al. 2014. Multiscale 3D manufacturing: combining thermal extrusion printing with additive and subtractive direct laser writing, in Proceedings of SPIE Vol. 9135: Laser Sources and Applications II. April 14, 2014, Brussels, Belgium. Bellingham: SPIE, 91350T. http://dx.doi.org/10.1117/12.2051520

[4] Jonušauskas, L., et al. 2015. Custom on demand 3D printing of functional microstructures, Lithuanian Journal of Physics 55(3): 227-236. http://dx.doi.org/10.3952/physics.v55i3.3151

[5] Jonušauskas, L.; Rekštytè, S.; Malinauskas, M. 2014. Augmentation of direct laser writing fabrication throughput for three-dimensional structures by varying focusing conditions, Optical Engineering, 53(12): 125102. http://dx.doi.org/10.1117/1.OE.53.12.125102

[6] Malinauskas, M., et al. 2016. Ultrafast laser processing of materials: from science to industry, Light: Science \& Applications 5: e16133. http://dx.doi.org/10.1038/lsa.2016.133 